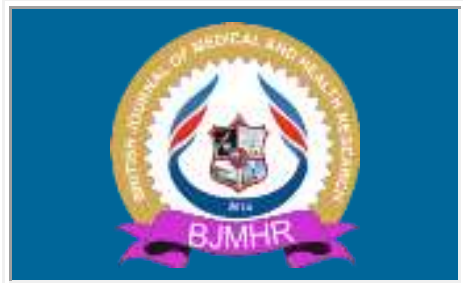

\title{
BJMHR
}

British Journal of Medical and Health Research Journal home page: www.bjmhr.com

\section{Association Between Childhood Seizures and Speech and} Language Impairment

\author{
Bharati Mehta $^{1}$, Anu Vyas ${ }^{2 *}$,Bharti Bhandari ${ }^{3}$, Manish Parakh ${ }^{2}$ \\ 1.AIIMS, Jodhpur \\ 2.SN Medical College, Jodhpur \\ 3.GIMS, Greater Noida, U.P.
}

\section{ABSTRACT}

A large group of children develop language impairment where an obvious cause for the same such as impaired hearing, mental retardation, cleft lip/palate, gross neurological or neuromuscular abnormalities affecting the speech apparatus, and autism cannot be found. This group is labeled as specific language impairment (SLI) The study aimed to assess the association between specific language impairment and childhood seizures. Out of 94 patients of SLI, about one fifth 19 (20.21\%) had history suggestive of seizures, of which 15 $(78.9 \%)$ were males and $4(21.05 \%)$ females. Majority $(57.9 \%)$ of patients had generalized tonic-clonic seizures $(n=11)$, in $21 \%$ of patients the picture was that of myoclonic seizures $(n=4)$, in another $15.8 \%(n=3)$ history of status epilepticus was documented. Associated risk factors of SLI such as family history of seizures, prenatal, perinatal and postnatal factors were also studied. The current study observed a very high association of seizures in this cohort of children with specific language impairment. Since SLI has a multifactorial etiology, seizures per se could not cause these disorders. Prenatal and perinatal insults, along with other risk factors may account for development of SLI. However, presence of seizures, particularly in infancy should be considered a warning signal to screen such children for development of specific language impairment.

Keywords: EEG, Seizures, Specific language impairment. 


\section{INTRODUCTION}

Language is one of the fundamental basis of human intelligence and a key part of human culture. Any insult to the language areas of the developing brain may result in impairment, depending upon the area of the brain bearing the brunt of the pathology.

Determining the prevalence of expressive language disorders at 24 months of age, based on a cut-off level of fewer than 50 words yields a range of $10 \%$ to $20 \%$ with boys having higher rates of delay than girls (1). These disorders are important because they interfere with the child's ability to communicate and learn (2).

Common causes of language disorders include impaired hearing, mental retardation, cleft lip/palate, gross neurological or neuromuscular abnormalities affecting the speech apparatus, and autism. One large group comprises of children in whom speech and language abnormality is not a result of any of these aforementioned causes and this group of children is designated as having specific language impairment (SLI).

Plausible risk factors for specific developmental language disorders are male gender, low birth weight, prematurity, neurometabolic disorders, structural abnormalities of brain, neurogenetic disorders, history of seizures, family history of seizures, etc. However, not all the children who develop perinatal problems, develop specific developmental language disorders. Seizures, particularly during infancy, constitute the commonest clinical manifestations of neurological dysfunction in an infant (3-5), it is therefore reasonable to assume that seizures may later affect the speech \& language development.

The study aimed to assess the association between developmental speech \& language impairment and childhood seizures .

\section{MATERIALS AND METHOD}

The present study was conducted in the Department of Physiology, Dr. S.N. Medical College, Jodhpur in association with Pediatric Neurology Clinic in Department of Pediatrics. Children between age group 2-8 years presenting predominantly with speech and language impairment were screened for participation in the study. Those having global motor developmental delay, head injury, gross hearing impairment, structural abnormalities of lips, tongue palate such as cleft lip/palate and autism were excluded from the study. These criteria corresponded closely with the definition of SLI.

Finally, 94 patients were selected having specific developmental language disorder, of which 18 were girls and 76 boys. After obtaining the permission from institutional ethical committee, an informed consent from parents of children was obtained for the study after explaining them clearly, the purpose of the study. A detailed history of the patients was then obtained from the 
parents which included prenatal, perinatal, postnatal pre/perinatal history, family history for any speech and language disorders \& seizures and neurodevelopmental history was obtained. Nineteen out of 94 children gave history of seizures. These children were then subjected for a detailed neurologic examination by a pediatric neurologist.

In all these patients, standard video EEG was performed for a minimum of 40 minutes to capture both wakefulness and sleep in stage II NREM. Bipolar and referential montages were used as per 10:20 international system of recording. The EEG recordings were reviewed by a trained pediatric neurologist and electrophysiologist to identify epileptiform or other changes, if any.

According to the International Federation of Societies for Electroencephalography and clinical Neurophysiology (IFSECN), epileptiform waves or activity, have been defined as "distinctive waves or complexes, distinguished from background activity, and resembling those recorded in a proportion of human subjects suffering from epileptic disorders"(6).

\section{RESULTS AND DISCUSSION}

\section{Epidemiological characteristics}

The final cohort constituted 94 children in the age group of 2 to 8 years, out of which 76 $(80.86 \%)$ were males and $18(19.14 \%)$ females; a sex ratio of $4.2: 1$. This is in congruence with the study performed by Shriberg, Tomblin, and McSweeny 1999 (4). Male is considered as an important risk factor for language-related changes, because of the slow maturation of the nervous system among boys, which leads to more vulnerability to changes in general, and also by the influence of testosterone, which stops cell death and makes the proper connections difficult, so it can have a negative effect on the development of areas involved with the language skills of these participants (5).

The mean age of whole cohort was $50.3 \pm 19.7$ months and was $49.3 \pm 19.5$ months in female children $(\mathrm{n}=18)$ and $51.3 \pm 20.0$ months in male children.

\section{Seizures}

About one fifth 19 (20.21\%) out of 94 children had history suggestive of seizures, of which 15 $(78.9 \%)$ were males and $4(21.05 \%)$ females. (Table 1$)$

Thus an unexpectedly high frequency of previous history of seizures is observed in the present cohort as compared to normal population where the prevalence ranges from 3.2-5.5/1,000 in developed countries and 3.6-44/1,000 in underdeveloped countries (7).

Six $(31.5 \%)$ developed seizures during their neonatal period; 9 (47.3\%) had their first seizure during their second month to $12^{\text {th }}$ month of life, and rest $4(21 \%)$ in their second year of life. (Table 1) 
Table 1 Predisposing characteristics for SLI in seizure patients

\begin{tabular}{lll}
\hline Age of onset of seizures & Neonatal (<1 month) & 6 \\
& Infantile (1-12 months) & 9 \\
& Childhood (>1 year) & 4 \\
Types of seizures & Generalized Tonic-Clonic & 11 \\
& Myoclonic & 4 \\
& Status Epilepticus & 3 \\
& Undefined & 1 \\
EEG changes & Normal EEG & 5 \\
& Generalized epileptiform & 4 \\
& Focal epileptiform & 3 \\
& Multifocal epileptiform & 3 \\
& Generalized \& Multifocal epileptiform & 2 \\
Other-associated & Non- epileptiform & 2 \\
conditions & Positive family history & 5 \\
& Gender (M:F) & $3.75: 1$ \\
& Asphyxia at birth & 3 \\
& LSCS (delayed labor, maternal diabetes) & 2 \\
& Hyperactivity & 9 \\
& Delayed milestones & 11 \\
\hline
\end{tabular}

Neonatal seizures may be indicative of a subtle neurodevelopmental vulnerability which may manifest later in life as specific learning difficulties or poor social adjustment (8). Higher proportion (22.2) of females had history of seizures compared to that of males and this difference was found statistically significant with a $\mathrm{p}$ value of 0.04 . (Table 1,2). In majority (57.9\%) of patients the seizures were generalized tonic-clonic type $(\mathrm{n}=11)$, in $21 \%$ of patients the picture was that of myoclonic seizures $(n=4)$, in another $15.8 \%(n=3)$ history of status epilepticus was there which led eventually to encephalopathy. Hypocalcemia was documented in one child presenting with myoclonic seizures. The authors could not get documented details of the seizures of one child nor did the parents of child recall it. (Table 1)

Table 2 Gender wise distribution of patients according to status of history of seizures.*Figures in parenthesis indicate percentage

\begin{tabular}{llll}
\hline Sex & \multicolumn{2}{l}{ History of seizures } & Total \\
& Yes & No & \\
\hline Male & $15(19.7)$ & $61(80.3)$ & $76(100)$ \\
Female & $4(22.2)$ & $14(77.8)$ & $18(100)$ \\
Total & $19(20.2)$ & $75(79.8)$ & $94(100)$ \\
$\chi^{2}=4.22, \mathrm{df}=1, \mathrm{p}$ value $=0.04$ & \\
\hline
\end{tabular}

Seizures are known to cause hypoxic brain injury. Surprisingly little research on the influence of fetal distress or neonatal seizures on SLI have been conducted. Most studies have focused on the influence of birth weight, gestational age, and infant feeding on later psychosocial difficulties. One study of long-term outcome after neonatal seizures in "normal" survivors 
found that over half of the children studied had social adjustment difficulties in their teenage years (8).

All 19 patients developed seizures during first 2 years of their life. This corroborates with findings of Jacobs and colleagues who found that social problems are more likely to be present if the brain injury occurred prior to 2 years of age (9).

\section{Family history of seizures}

Fifteen $(16 \%)$ of 94 children of this cohort had family history of seizures. Of which 12 (15.8 $\%)$ were boys and $3(16.7 \%)$ were girls.

Five out of 19 (26\%) children with seizures showed family history of seizures. In many studies on identification of risk factors in children with speech disorders, it was proposed that family history is a potential risk factor for development of speech and language impairment $(6,10)$. This association is not only because of genetic predisposition but also because of the common environment, the family members share. Many childhood epilepsy syndromes have a familial predisposition, and the genetic bases for several disorders have been described (11).

\section{EEG Changes}

Fourteen out of $19(73 \%)$ patients with seizures showed an abnormal EEG as compared to 31 (41\%) of 75 patients without seizures. This difference was also found statistically significant $(\mathrm{p}=0.01)$. In patients with abnormal EEG, higher proportion $(31.1 \%)$ of the patients had history of seizures compared to patients with normal EEG (10.2\%). This difference was also found statistically significant $(\mathrm{p}=0.01$, Table 3 )

Table 3: Distribution of patients showing abnormal EEG with history of Seizures *Figures in parenthesis indicate percentage

\begin{tabular}{llll}
\hline Status of EEG & \multicolumn{2}{l}{ History of seizures } & Total \\
& Yes & No & \\
\hline Normal & $5(10.2)$ & $44(89.8)$ & $49(100.0)$ \\
Abnormal & $14(31.1)$ & $31(68.9)$ & $45(100.0)$ \\
Total & $19(20.2)$ & $75(79.8)$ & $94(100.0)$ \\
$\chi^{2}=6.35, d f=1, p$ value=0.01 & & \\
\hline
\end{tabular}

Five patients with seizures showed a normal EEG; 12 showed abnormalities labeled as epileptic: inter-ictal discharges, focal spikes or paroxysmal bursts of spike/ wave activity. Another 2 showed abnormalities, which were not specifically epileptic such as intermittent sharp waves from P3 and sudden appearance of theta activity in one and slow for age \&paucity of normal features of stage II NREM sleep in another patient.

These results are in congruence with a study that presented case series of nine children having severe disturbances of language function. Seizures occurred in seven of them and all nine had EEG abnormalities. However, there was no apparent correlation between the seizures, the EEG 
changes and the aphasia (12). The present study is also in agreement with a study by Aina et al which showed a positive connection among SLI, seizures, hyperactivity etc (13).

Previous studies have consistently shown seizure to be a major complication among children with developmental speech disorders (14).

Some studies have also demonstrated that abnormal electroencephalographic recordings without clinical seizures can be observed (15).

\section{Other Associations (Table 1)}

\section{LSCS:}

Out of 19 children having history of seizures, 2 were born by LSCS; the indication of which was failed progress in one and maternal diabetes in other. Rest 17 children were born of normal vaginal delivery. One child was born at home. Complications in labour and delivery are some of the maternal risk factors for developmental speech and language delay in children (16).

\section{Asphyxia:}

Three children developed asphyxia soon after their birth, however resuscitation was not required in any of them. Neonatal hypoxia can predispose a child to seizures as well as brain ischemia and if the ischemia damages the language areas, the child may develop speech and language impairment.

\section{Delayed milestones:}

Parents of 11 children reported delayed milestones of development along with speech \& language impairment. Minor neurodevelopmental abnormalities, genetically transmitted or acquired during the pre- or perinatal period, may create vulnerability towards SLI. This hypothesis underlines the importance of prevention and early detection of SLI when identifying vulnerable subjects (15).

\section{Hyperactivity:}

Nine of 19 children were very hyperactive \& fidgety. They cannot follow instructions or concentrate. Complications as seizures, hyperactivity possibly reinforce the previously subtle brain damage among children. The need for preventive health care is therefore emphasized (13).

\section{CONCLUSION:}

The current study observed a very high association of seizures in this cohort of children with specific language impairment. Others studies have also found such association $(17,18)$. Seizures might cause language disorders by either directly damaging language areas or by causing abnormal brain development. It is well known that SLI has a multifactorial etiology, so seizures per se could not cause these disorders. Prenatal and perinatal insults, along with other risk factors account for development of these disorders. However, presence of seizures, 
particularly in infancy should be considered a warning signal to screen such children for development of specific language impairment.

\section{ACKNOWLEDGEMENT:}

The authors are grateful to the children who participated and their parents who consented for the study.

\section{REFERENCES:}

1. Rescorla L, Alley A. Validation of the language development survey (LDS): a parent report tool for identifying language delay in toddlers. J Speech Lang Hear Res JSLHR. 2001; 44: 434-45.

2. Howlin P, Mawhood L, Rutter M. Autism and developmental receptive language disorder--a follow-up comparison in early adult life. II: Social, behavioural, and psychiatric outcomes. J Child Psychol Psychiatry. 2000; 41: 561-78.

3. Eun S, Lee JM, Yi DY, Lee NM, Kim H, Yun SW, Lim I, Choi ES, Chae Assessment of the association between Apgar scores and seizures in infants less than 1 year old. Seizure. 2016 ; 37: 48-54.

4. Shriberg LD, Tomblin JB, McSweeny JL. Prevalence of speech delay in 6-year-old children and comorbidity with language impairment. J Speech Lang Hear Res JSLHR. 1999 ; 42: 1461-81.

5. Silva GMD, Couto MIV, Molini-Avejonas DR, Silva GMD, Couto MIV, MoliniAvejonas DR. Risk factors identification in children with speech disorders: pilot study. CoDAS. 2013; 25: 456-62.

6. International Federation of Societies for Electroencephalography and Clinical Neurophysiology (IFSECN). A glossary of terms most commonly used by clinical Electroencephalographers, Electroencephalography and Clinical Neurophysiology 1974; $37: 538$ - 548 .

7. Camfield P, Camfield C. Incidence, prevalence and aetiology of seizures and epilepsy in children. Epileptic Disord Int Epilepsy J Videotape. 2015; 17: 117-23.

8. Temple CM, Dennis J, Carney R, Sharich J. Neonatal seizures: long-term outcome and cognitive development among “normal” survivors. Dev Med Child Neurol. 1995; 37 : $109-18$.

9. Jacobs R, Harvey AS, Anderson V. Executive function following focal frontal lobe lesions: impact of timing of lesion on outcome. Cortex J Devoted Study Nerv Syst Behav. 2007; 43: 792-805. 
10. Chaimay B, Thinkhamrop B, Thinkhamrop J. Risk factors associated with language development problems in childhood--a literature review. J Med Assoc Thail Chotmaihet Thangphaet. 2006; 89: 1080-6.

11. Arnold ST, Dodson WE. Epilepsy in children. Baillieres Clin Neurol. 1996; 5: 783802.

12. Echenne B, Cheminal R, Rivier F, Negre C, Touchon J, Billiard M. Epileptic electroencephalographic abnormalities and developmental dysphasias: a study of 32 patients. Brain Dev. 1992; 14: 216-25.

13. Aina OF, Ogun OC, Ladapo HT, Lesi FE, Famuyiwa OO. Clinical neuropsychiatric correlates and EEG findings among children with developmental disorders in Lagos, Nigeria. Afr J Psychiatry. 2008; 11: 123-7.

14. Wing L. The autistic spectrum. Lancet. 1997; 350: 1761-6.

15. Lévy-Rueff M, Bourgeois M, Assous A, Beauquier-Maccota B, Boucheron E, Clouard C, Donde S, Eostini O, Pinot P, Mosser A, et al. Abnormal electroencephalography results and specific language impairment: towards a theoretical neurodevelopmental model?. L'Encéphale. 2012; 38: 318-28.

16. Delgado CE, Vagi SJ. Early risk factors for preschool speech and language impairments. In: PrQceedings of the international Conference on infant Studies. 2004; 571-89.

17. Dalby, M. A. Aetiological studies in language retarded children. Neuropadiatrie, 1977; 8: 499-500

18. Mellor, D. H. A neurological study of children attending a special residential school for children with severe speech and language disorders. Neuropediatrics, 1981; 12: 436-437

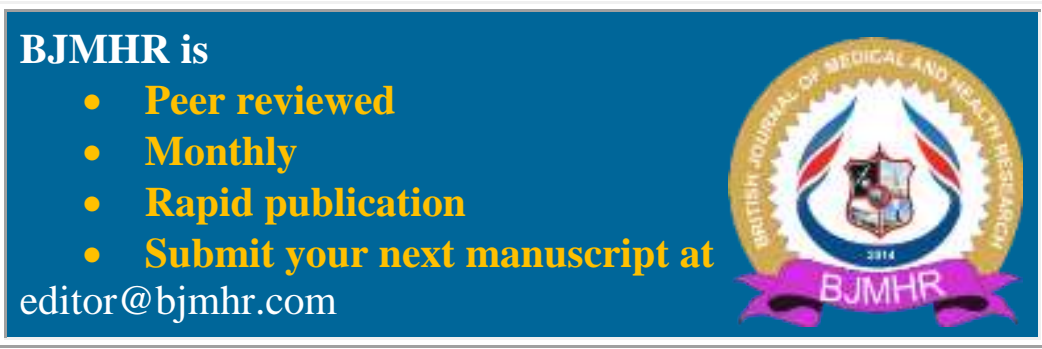

